\title{
Confini, migrazioni, cittadinanza ${ }^{1}$
}

\section{Sandro Mezzadra}

Università di Bologna. Facoltà di Scienze Politiche

\begin{abstract}
La proliferazione dei confini, intesa come una loro scomposizione e ricomposizione, costituisce uno dei risultati piú immediati dei processi di globalizzazione; partendo da questa affermazione, l'autore riflette sulla nuova natura che i confini stanno assumendo, in particolare con riferimento ai movimenti migratori contemporanei, al loro aspetto di «turbolenza» e ai processi di trasformazione e ri-definizione della cittadinanza a questi connessi.
\end{abstract}

Parole chiave: confini, migrazioni, cittadinanza, globalizzazione.

\section{Abstract. Borders, migration and citizenship}

The proliferation of boundaries, i.e. their constant fall and re-composition, is one of the most evident outcomes of globalisation. Setting off from this statement, the author explores the new nature that boundaries are assuming, with a particular reference to contemporary migratory flows, their «turbulent» aspects and associated processes of transformation and re-definition of citizenship.

Key words: boundaries, migrations, citizenship, globalisation.

\section{Sommario}

\section{Prologo 4. Migrazioni globali}

2. Un concetto classico di confine $\quad 5$. Border studies

3. S/confinamenti 6. Conflitti di cittadinanza

1. Il testo riproduce, conservandone la forma di esposizione orale, la relazione da me presentata al convegno di Bolzano. Una precedente versione è stata pubblicata in Scienza \& Politica, n. 30, 2004. 


\section{Prologo}

Una mostra presentata a Modena, durante il Festival di filosofia dello scorso anno, si intitolava "Border devices». Nell'introdurla, gli organizzatori scrivevano che la proliferazione dei confini, il loro prismatico scomporsi e ricomporsi, costituisce «'altro lato della globalizzazione». E aggiungevano: «il sogno di uno spazio totalmente fluido e attraversabile è forse l'ultima utopia del Ventesimo secolo. Il carattere liscio che sarebbe proprio dello spazio contemporaneo si dissolve a uno sguardo più ravvicinato. Uno dei risultati più immediati dei movimenti e delle interconnessioni globali pare consistere piuttosto in una proliferazione di confini, sistemi di sicurezza, checkpoints, frontiere fisiche e virtuali. È un fenomeno che possiamo osservare sia al livello micro dei territori in cui ci muoviamo ogni giorno, sia al livello macro dei flussi globali: i confini, in effetti, sono tutt' intorno a noi. Sono confini convenzionali e geografici, astratti e reali, assunti come ovvi e contestati. Uno sguardo d'insieme a questa combinazione di flussi (di persone, merci, idee...) e di restrizioni su un dato territorio rivela la complessità di identità individuali e collettive che sono, al tempo stesso, costruite e fratturate dall'esperienza dell'attraversamento dei confini» ${ }^{2}$.

Sarà bene tenere sullo sfondo, nel corso dell'intera nostra discussione sui confini, questo riferimento alle identità fratturate dall'esperienza dell'attraversamento dei confini. Una delle sale in cui si articolava la mostra modenese riproduceva un'installazione video in due ambienti prodotta in occasione di Documenta XI a Kassel, dedicata a ricostruire la storia del tragico naufragio avvenuto nella notte di Natale del 1996 nelle acque del Canale di Sicilia, nel corso del quale perirono 283 migranti tamil, pakistani e indiani. L'attraversamento dei confini, oggi, è anche questo: un drammatico campo di tensione in cui spesso la ricerca di una vita migliore si infrange sulla catastrofe della morte. Non lo dimentichiamo, neppure quando, come è necessario in un convegno di studi, il nostro ragionamento viene svolgendosi sul piano dell'astrazione teorica e nel linguaggio spesso rarefatto delle molte discipline che affrontano il tema dei confini.

\section{Un concetto classico di confine}

L'obiettivo della mia relazione consiste nel proporre alcune osservazioni preliminari sulla nuova natura che i confini assumono nel nostro tempo, con particolare riferimento ai movimenti migratori e al rapporto che essi intrattengono con le trasformazioni della cittadinanza. Lo sfondo della relazione è costituito da un insieme di ricerche sui movimenti migratori contemporanei ${ }^{3}$ e, al tempo stesso, da un lavoro di precisazione della storia concettuale della cittadinanza

2. Cfr. <www.multiplicity.it>.

3. Mezzadra, S. Diritto di fuga. Migrazioni, cittadinanza, globalizzazione. Verona: Ombre corte, 2001; Mezzadra, S. (a cura di). I confini della libertà. Per un'analisi politica delle migrazioni contemporanee. Roma: Derive Approdi, 2004. 
moderna europea e occidentale che ha posto in evidenza il rilievo costitutivo, all'interno di questa storia, di quelli che ho altrove definito i confini della cittadinanza $a^{4}$. E si tratta di confini la cui valenza va oltre la dimensione che possiamo definire per comodità "geopolitica», investendo l'antropologia politica implicita nel moderno discorso della cittadinanza, ovvero i modi in cui storicamente l'individuo è stato immaginato e costruito come cittadino 5 .

Il punto di partenza che vorrei proporre per le osservazioni che seguono è un concetto che possiamo definire classico di confine ${ }^{6}$, quale emerge congiuntamente, con particolare nettezza, dai contemporanei sviluppi della dottrina generale dello Stato (G. Jellinek) e della "geografia politica» (F. Ratzel) tra Otto e Novecento in Germania. "La connessione tra territorio e Stato» si intitola significativamente la prima parte del grande trattato di geografia politica pubblicato da Ratzel nel 1897.

«Ogni Stato», si legge proprio all'inizio dell'opera di Ratzel, «è una porzione di umanità e una porzione di territorio. L'uomo non è pensabile senza la terra, e tanto meno lo è la più insigne opera dell'uomo sul nostro pianeta, ovvero lo Stato» ${ }^{7}$.

È lo stesso Ratzel a sottolineare la convergenza di questa immagine dello Stato con la definizione della sovranità come jus territoriale elaborata negli stessi anni dalla dottrina generale dello Stato ${ }^{8}$. Nella Allgemeine Staatslehre di Georg Jellinek (1900), il carattere unitario del territorio dello Stato è —insieme alla concomitante unitarietà del popolo dello Stato e del potere dello Stato- uno dei tre elementi essenziali che concorrono alla stessa definizione dello Stato. In questo quadro, la definizione del confine è relativamente poco problematica: esso è l'astrazione che consente di delimitare il processo dinamico di espansione della forma di vita politica di un popolo9 ${ }^{9}$, o il limite dell'ambito territoriale di validità del potere dello Stato (Jellinek). All'interno di una diversa

4. Cfr. ad es. Mezzadra, S. (a cura di). Cittadinanza. Ordine, soggetti, diritti. Bologna: Clueb, 2004.

5. SANtoro, E. Autonomia individuale, libertà e diritti. Una critica dell'antropologia liberale. Pisa: ETS, 1999; CosTA, P. Civitas. Storia della cittadinanza in Europa. 4 vol. Roma, Bari: Laterza, 1999-2001; MEZZADRA, S. Immagini della cittadinanza nella crisi dell'antropologia politica moderna. Gli studi postcoloniali. In: GHERARDI, R. (ed.). Politica, consenso, legittimazione. Trasformazioni e prospettive. Roma: Carocci, 2002, p. 85-100.

6. Cfr. al riguardo PrescotT, J.R.V. Political Frontiers and Borders. London: Allen \& Unwin, 1987.

7. Ratzel, F. Politische Geographie (1897), 3. Aufl., durchgesehen und ergänzt von E. Oberhummer, Oldenbourg, München, Berlin, 1923, p. 2. Tra gli studi recenti dedicati a Ratzel, si segnala il saggio di FARINELLI, F. «Friedrich Ratzel and the Nature of (Political) Geography». Political Geography, 19, 2000, p. 943-955. Ricco di riferimenti a Ratzel è poi il recente volume di CavalletTI, A. La città biopolitica. Mitologie della sicurezza. Milano: Bruno Mondadori, 2005.

8. Cfr. Ratzel, F. Politische Geographie. Cit., p. 3.

9. Ivi, p. $384 \mathrm{~s}$. 
tradizione, ma in consonanza con questa linea argomentativa, Lord Curzon affermava nel 1908 che «'integrità dei confini è la condizione di esistenza dello Stato", il segno visibile della tenuta di quella distinzione fra interno ed esterno che sola poteva garantire l'ordine e la pace. Lord Curzon si affrettava infatti ad aggiungere che i confini sono «la lama di rasoio su cui sono sospese le questioni moderne della guerra e della pace» ${ }^{10}$.

$\grave{E}$ appena il caso di notare, per venire al tema direttamente al centro della relazione, come l'architettura geopolitica e giuridica organizzata attorno al concetto che ho definito classico di confine abbia costituito la cornice al cui interno si è svolta la storia delle migrazioni in Europa tra il XIX e il XX secolo. La tenuta del confine, e della secca distinzione tra interno ed esterno che esso garantiva, è stata in particolare la condizione che ha consentito il prendere forma di precisi sistemi migratori e di una relativamente ordinata geografia delle migrazioni internazionali. Si potrà obiettare, e legittimamente, che sotto il profilo storiografico questo presupposto ha spesso condotto a una rappresentazione pacificata e idilliaca delle migrazioni in Europa - a dimenticare quello che Saskia Sassen ha definito "il cono d'ombra della storia d'Europa», in cui "vi sono masse di individui deportati, sradicati ed errabondi che vivono in terra straniera, in paesi che non riconoscono loro alcuna "appartenenza»" ${ }^{11}$. Ma per una ricostruzione "tipologica» come quella a cui qui si tende, orientata a far emergere le peculiarità della situazione contemporanea, è forse più rilevante osservare come quell'architettura abbia cominciato a vacillare laddove più problematico appariva il presupposto di una co-appartenenza di Stato e territorio: in territori solcati da linee di frattura «nazionali», «etniche» e «linguistiche», come ad esempio le province prussiane orientali negli anni ' 90 dell'Ottocento ${ }^{12}$, o nella crisi dei profughi successiva alla prima guerra mondiale, secondo la classica analisi proposta da Hannah Arendt in Le origini del totalitarismo.

Vale la pena del resto di sottolineare, e sia Ratzel sia Jellinek lo riconoscono, che il principio dell' unitarietà territoriale dell'ordinamento conosceva una assai significativa eccezione — una vera e propria anomalia - a proposito della posizione delle colonie: nella prospettiva di Ratzel, infatti, l'espansione coloniale rappresentava sì la conferma della «dinamicità» dell'organismo statuale, ma costituiva al tempo stesso un'incrinatura della sua compattezza proprio per il venir meno della continuità territoriale; nella prospettiva di Jellinek, essa introduceva una potenziale crepa, per via del necessario riconoscimento di una pluralità di ordinamenti e di posizioni soggettive, nell'unitarietà dello spazio giuridico organizzato dallo Stato ${ }^{13}$.

10. Lord Curzon. Frontiers [The Romanes Lecture 1907]. Oxford: Clarendon Press, 1908, p. 7.

11. SASSEN, S. Migranti, coloni, rifugiati. Dall'emigrazione di massa alla fortezza Europa (1996). Milano: Feltrinelli, 1999, p. 18.

12. Mezzadra, S. Diritto di fuga. Cit., cap. 1.

13. Mezzadra, S.; Rigo, E. «Diritti d'Europa. Una prospettiva postcoloniale sul diritto coloniale». In: MAZZACANE, A. (a cura di). Oltremare. Diritto e istituzioni dal colonialismo all'età postcoloniale. Napoli: Liguori, 2005. In corso di pubblicazione. 


\section{S/confinamenti}

Riprendendo e svolgendo in tutt'altro contesto, quale ad esempio quello definito dallo sviluppo negli ultimi anni dei postcolonial studies, alcune indicazioni di Carl Schmitt (Il nomos della terra, 1950), possiamo affermare che l'architettura a cui si è fatto riferimento si fondava, come suo elemento costitutivo e dunque secondo un rapporto non riducibile a quello tra «interno» ed «esterno», sull'esistenza di quello che proporrei di chiamare un "metaconfine»: ovvero quello che divideva le terre europee prima, e «occidentali» dopo, dalle terre aperte alla conquista coloniale. In questo senso va a mio parere interpretato quanto ha scritto Etienne Balibar, e cioè che «l'Europa è il punto da cui sono partite, sono state tracciate dappertutto nel mondo le linee di confine, perché essa è la terra natale del concetto stesso di confine», e che dunque il problema dei confini dell'Europa è sempre coinciso con quello dell'organizzazione politica dello spazio mondiale ${ }^{14}$.

Due conseguenze di particolare importanza ne derivano: in primo luogo, si apre qui un campo di studio e ricerca (a cui sta ad esempio lavorando a Calcutta il gruppo coordinato da Ranabir Samaddar ${ }^{15}$ ) sulla funzione del confine all'interno del mondo coloniale (e sulla sua importanza nel "governare» $\mathrm{i}$ movimenti più o meno coatti di popolazione che furono uno degli elementi essenziali del dominio coloniale medesimo); in secondo luogo, se assumiamo in via di ipotesi che il presente sia caratterizzato dal venir meno del «metaconfine» di cui si è detto, si guadagna una prospettiva di indagine particolarmente interessante proprio sui movimenti migratori: da una parte si possono istituire inediti paralleli tra questi ultimi e lo sviluppo dei movimenti anti-coloniali nel corso del Novecento (per via della sfida che questi movimenti hanno portato al «metaconfine» coloniale); dall'altra si apre la possibilità che alcuni disjecta membra del colonialismo (la distinzione tra citizen e subject, ad esempio) si riproducano all'interno di quelle che un tempo erano le metropoli.

Da questo punto di vista, è possibile sviluppare in modo un poco più articolato l'affermazione da cui siamo partiti, l'idea cioè che la proliferazione dei confini costituisca l'«altro lato della globalizzazione». La tesi che si può sostenere è che la globalizzazione non sia certo contraddistinta dalla fine dei confini, ma piuttosto dalla crisi di quella connessione di Stato e territorio che abbiamo visto costituire il presupposto concettuale della definizione «classica» del confine. Il confine non separa più univocamente lo spazio della "città» dal suo esterno, ma si scompone prismaticamente, da una parte riproducendosi all'interno della città stessa e dall'altra proiettandosi al suo esterno. Space is out of joint, come riconoscono gli stessi geografi più avveduti ${ }^{16}$. Al contempo,

14. Balibar, E. Le crainte des masses. Politique et philosophie avant et après Marx. Paris: Galilee, 1997 , p. 382 e 387 s.

15. Di R. Samaddar si veda la recente raccolta di saggi, The Politics of Dialogue: Living Under the Geopolitical Histories of War and Peace. Aldershot: Ashgate, 2004.

16. Neve, M. Itinerari nella geografia contemporanea. Roma: Carocci, 2004, p. 27 s. (ma cfr. anche, specificamente sul tema del confine, p. 38 s.). 
l'univocità della definizione "geopolitica» del confine appare messa in discussione, e altre valenze del concetto - da quella culturale a quella simbolica a quella "cognitiva» ${ }^{17}$ — vengono in primo piano nello stesso operare politico del confine.

\section{Migrazioni globali}

La mia convinzione è che i movimenti migratori contemporanei permettano di precisare la tesi appena presentata, mostrando al contempo l'intensità delle tensioni e dei conflitti che sono in gioco in questo doppio movimento di scomposizione e di ricomposizione dei confini. Pur senza trascurare gli elementi di continuità che è possibile individuare tra i movimenti migratori degli ultimi anni e le migrazioni di un secolo fa, è necessario da questo punto di vista accentuare analiticamente gli altrettanto indiscutibili elementi di novità che caratterizzano $\mathrm{i}_{\text {primi }}{ }^{18}$. Le migrazioni contemporanee appaiono infatti caratterizzate da una moltiplicazione dei modelli migratori, da una forte accelerazione dei flussi, da un aumento di complessità della loro composizione (ad esempio con un forte incremento della partecipazione femminile) e da una crescente imprevedibilità delle loro direzioni ${ }^{19}$.

Quel che sempre più contraddistingue i movimenti migratori, secondo l'azzeccata definizione di uno studioso australiano, è la loro turbolenza ${ }^{20}$. È un elemento che balza agli occhi immediatamente non appena si tenti di ricostruire la geografia degli spostamenti contemporanei di popolazione: ancora nelle condizioni del secondo dopoguerra, ad esempio, era tutto sommato abbastanza agevole individuare i flussi dominanti, con aree di partenza e di destinazione stabili che definivano, come si è detto in precedenza, precisi «sistemi migratori» ${ }^{21}$. Oggi, al contrario, «i flussi vanno dappertutto», e, come è stato notato di recente, ogni tentativo di «dare una rappresentazione grafica» del fenomeno migratorio appare votato allo scacco, «a meno che non si voglia raffigurare una sorta di piatto di spaghetti» ${ }^{22}$.

17. Cfr. Zanini, P. Significati del confine. I limiti naturali, storici, mentali. Milano: Bruno Mondadori, 1997 e Tagliagambe, S. Epistemologia del confine. Milano: Il Saggiatore, 1997.

18. Cfr. Gozzini, G. «Migrazioni ieri e oggi: un tentativo di comparazione». Passato e presente, XXII (2004), n. 61, p. 35-63.

19. Castles, S.; Miller, M. J. The Age of Migration. International Population Movements in the Modern World. New York: The Guilford Press, $2003^{3}$. Per due sobrie analisi comparative dei movimenti migratori di «ieri» e di «oggi», cfr. GozZINI, G. «Migrazioni ieri e oggi: un tentativo di comparazione». Passato e presente, 22, 2004, p. 35-63 ed Morawska, E. «Immigrati di ieri e di oggi in Europa e fuori: insediamento e integrazione». In: CAPONIO, T.; Colombo, A. (a cura di). Migrazioni globali, integrazioni locali. Bologna: Il Mulino, 2005, p. 23-85.

20. Papastergiadis, N. The Turbolence of Migration. Globalization, Deterritorialization und Hybridity. Cambridge: Polity Press, 2000.

21. Cfr. ad es. SASSEN, S. Migranti, coloni, rifugiati. Cit., p. 95 s.

22. Macioti, M. I.; Pugliese, E. L'esperienza migratoria. Immigrati e rifugiati in Italia. Roma, Bari: Laterza, 2003, p. 17. 
La ricerca internazionale sulle migrazioni, conseguentemente, registra sempre più spesso la crisi dei modelli «idraulici» di interpretazione delle migrazioni stesse, centrati univocamente, sia nelle versioni neoclassiche sia nelle versioni neomarxiste, sui fattori di "attrazione» e di "spinta» (push and pull) che determinerebbero la scelta migratoria ${ }^{23}$ : sempre più emerge l'irriducibilità dei movimenti migratori agli schemi esplicativi fondati su elementi «oggettivi», o meglio l'eccedenza dei comportamenti soggettivi che in essi si manifestano rispetto alle pur evidenti motivazioni appunto «oggettive» delle migrazioni. E così, mentre sul piano analitico un'attenzione crescente viene ad esempio riservata dalla cosiddetta new economics of migration al ruolo delle reti familiari e "comunitarie» nel determinare tutte le fasi del processo migratorio, anche sotto il profilo delle politiche migratorie l' «utopia» del pieno controllo e del governo assoluto dei "flussi» lascia spazio ad approcci come il cosiddetto actors in chain approach, che punta a fronteggiare in modo contingente e "opportunistico» il margine di imprevedibilità a cui fanno riferimento concetti come «eccedenza» $\mathrm{e}$ "turbolenza» 24 .

\section{Border studies}

È sullo sfondo di queste nuove caratteristiche dei movimenti migratori che occorre porre la questione del rapporto che essi intrattengono con il confine, di cui occorrerà al contempo indicare almeno alcune delle nuove caratteristiche prima sinteticamente accennate. Un contributo particolarmente rilevante da questo punto di vista, guardando al dibattito internazionale sul tema, è quello offerto dallo sviluppo dei border studies statunitensi, e in particolare dai molti lavori di ricerca (etnografici, geografici, sociologici, giuridici, etc.) sulla frontiera tra Messico e Stati uniti. Questi lavori ${ }^{25}$ hanno insistito in particolare, per dirla in estrema sintesi, sulla funzione di ibridazione che l'esperienza della frontiera da parte dei migranti messicani e dei chicanos determina, consentendo il continuo «spiazzamento» dei confini identitari. Come ha scritto José David Salvídar:

[...] il confine tra Stati uniti e Messico [è] un paradigma dell'attraversamento, della circolazione, della mescolanza materiale, e della resistenza ${ }^{26}$.

23. Rinvio a questo proposito a MEZZADra, S. «Capitalismo, migrazioni, lotte sociali. Appunti per una teoria dell'autonomia delle migrazioni». In: MEZZADRA, S. (a cura di). I confini della libertà. Cit., p. 7-19.

24. Cfr., a proposito del caso europeo, Veenkamp, Th.; Bentley, T.; Buonfino, A. People Flow. Managing Migration in a New European Commonwealth (2003). <www.demos.co.uk/ peopleflow_pdf_media_public.aspx.>

25. Mi limito qui a ricordare, in luogo di molti, quello — per molti versi «fondatore»— di ANZALDÚA, G. Terre di confine/La frontera (1987). Bari: Palomar, 2000.

26. Saldívar, J. D. Border Matters. Remapping American Cultural Studies. Berkeley: University of California Press, 1997. 
Certo, è bene ricordare che proprio all'inizio del libro di Gloria Anzaldúa, che viene abitualmente indicato come punto di svolta nei border studies statunitensi, si può leggere che il confine, "questo luogo di contraddizioni»,

[...] non è un territorio confortevole per viverci»: «odio, rabbia e sfruttamento sono le caratteristiche preminenti di questo paesaggio ${ }^{27}$.

"Questa è la mia casa/questa sottile linea di/filo spinato», scriveva la scrittrice e poeta chicana, che aggiungeva subito dopo ritornando alla prosa:

[...] il confine tra Stati uniti e Messico es una ferida abierta dove il Terzo mondo si scontra con il primo e sanguina ${ }^{28}$.

E tuttavia la tesi di Anzaldúa secondo cui la frontiera costituisce «un terzo spazio", in particolare nella rappresentazione folcloristica e commercializzata che ne è stata offerta da parte di artisti come Guillermo Gómez-Peña, ha finito per nutrire una vera e propria nuova ortodossia nei border studies. E in questa forma è stata sottoposta negli ultimi anni a una crescente critica per i suoi tratti «estetizzanti» —in particolare, mette conto sottolinearlo, da parte di studiosi messicani ${ }^{29}$.

Fermo restando che, a mio giudizio, libri come quello di Anzaldua, oltre a essere letture straordinariamente affascinanti, colgono alcuni elementi di realtà ( $\mathrm{e}$ in particolare, in un contesto come quello italiano, rappresentano una salutare sfida all'essenzialismo culturale dilagante nel discorso scientifico e nel senso comune a proposito del «multiculturalismo»), si può raccogliere l'invito che viene da Pablo Vila, autore di fondamentali studi sul confine tra Messico e USA, a rivisitare la teoria della frontiera ponendo l'accento sulla sua ambivalenza, sulla dialettica, nella stessa esperienza dei migranti, tra border crossing e border reinforcing ${ }^{30}$.

Questa ambivalenza, che altrove ho cercato a mia volta di sottolineare sviluppando la distinzione fra "frontiera» e "confine» ${ }^{31}$, emerge molto chiaramente anche a proposito di uno dei campi di studio più innovativi degli ultimi anni nella ricerca sulle migrazioni, quello a cui fa cioè riferimento il concetto di transnazionalismo (che gioca del resto un ruolo sempre più importante negli studi storici, come dimostrano ad esempio i lavori di Donna Gabaccia e di Emilio Franzina sull'emigrazione italiana verso le Americhe). La tendenza dei movimenti migratori contemporanei a costruire e moltiplicare spazi sociali

27. AnZaldúa, G. Terre di confine. Cit., p. 21.

28. Ibidem, p. 29.

29. Cfr. ad esempio TABuenca Cóndoba, M. S. «Aproximaciones críticas sobre las literaturas de las fronteras». Frontera Norte, IX, 1997, 18, p. 85-110.

30. Cfr. in particolare VILA, P. "The Limits of American Border Theory». In: ViLA, P. (ed.). Ethnography at the Border. Minneapolis, London: Univeristy of Minnesota Press, 2003, p. 306-341.

31. Cfr. Mezzadra, S. Diritto di fuga. Cit., cap. 3. 
transnazionali, contribuendo al continuo rimescolamento della carta geografica del pianeta, non può certo essere negata, al pari delle potenzialità che questa tendenza contiene al suo interno per un ripensamento della cittadinanza: ma una lettura meramente estetizzante del transnazionalismo, come è stato ad esempio sottolineato da Ruba Salih ${ }^{32}$, mancherebbe di cogliere appunto la sua profonda ambivalenza, la riproduzione all'interno degli spazi transnazionali e proprio facendo leva sulla persistente vigenza dei confini, di vecchie e nuove gerarchie di classe e di genere ${ }^{33}$.

D'altro canto, per analizzare le metamorfosi del confine nel contesto dei processi di globalizzazione, non è indispensabile assumere come punto privilegiato di osservazione la frontiera tra Usa e Messico: proprio l'Europa costituisce infatti un eccellente caso di studio. Qui, tra l'accordo di Schengen e la convenzione di Dublino e poi nel contesto del processo di allargamento dell'Unione europea, ha preso forma, proprio intorno alla retorica del necessario contrasto dell' «immigrazione clandestina», un nuovo regime di controllo dei confi$\mathrm{ni}$, per molti aspetti paradigmatico ${ }^{34}$. $\mathrm{E}$ un regime flessibile e a geometria variabile, che assai più che a consolidare le muraglie di una "fortezza», e dunque a segnare una rigida linea di demarcazione fra il dentro e il fuori, sembra puntare a governare un processo di inclusione differenziale dei migranti. Il nuovo regime dei confini di cui sto parlando è un regime strutturalmente ibrido di esercizio della sovranità, alla cui definizione e al cui funzionamento concorrono gli Stati nazionali (in misura sempre meno esclusiva, ma mostrando proprio qui la propria persistenza nello scenario della "globalizzazione»), formazioni "postnazionali» come l'Unione europea, nuovi attori globali come l'International Organization for Migration, soggetti privati come le compagnie aeree e Organizzazioni non governative dalle finalità «umanitarie».

Ancora una volta in estrema sintesi, credo che si possa concordare con quanto ha scritto di recente una studiosa italiana che si è occupata a più riprese del problema:

[...] la progressiva deterritorializzazione dei confini esterni e interni della polis europea rende discontinuo il suo spazio giuridico e ammette una sovranità condivisa tra attori diversi, sia pubblici che privati ${ }^{35}$.

32. SaliH, R. Gender in Transnationalism. Home, Longing and Belonging Among Moroccan Migrant Women. London, New York: Routledge, 2003.

33. Si veda anche, ad es., Ong, A. Flexible Citizenship. The Cultural Logics of Transnationality. Durham, London: Duke University Press, 1999.

34. Cfr. ad es. WALTERS, W. «Mapping Schengenland: Denaturalizing the Border». Environment and Planning D: Society and Space, vol. 20, 2002, 5, p. 561-58. Ma si veda anche il recente volume di BECK, U.; GRANDE, E. Das kosmopolitische Europa. Frankfurt a.M.: Suhrkamp, 2004 , in cui la mobilità e la flessibilità dei confini è indicata come caratteristica fondamentale della stessa struttura istituzionale dell'Unione europea.

35. Rigo, E. «Ai confini dell'Europa. Cittadinanze post-coloniali nella nuova Europa allargata». In: Mezzadra, S. (ed.). I confini della libertà. Cit., p. 82. 
Per deterritorializzazione si deve intendere sia lo spostamento di funzioni tipiche del controllo dei confini ben al di là della linea di confine (si pensi solo a quello che sta accadendo in questi mesi in Libia, ma anche a quanto accade quotidianamente nel mediterraneo, attraversato da quelli che il Consiglio europeo del novembre dello scorso anno ha definito "confini virtuali» coincidenti in ultima istanza con le imbarcazioni che trasportano migranti), sia la disseminazione di quelle stesse funzioni all'interno dello spazio che il confine dovrebbe perimetrare (si pensi ai centri di detenzione per migranti in attesa di espulsione, presenti in quasi tutti i paesi europei). Più in generale, il confine prolunga la sua azione all'interno della citta anche da un altro punto di vista: assecondando la tendenza alla produzione di una pluralità di posizioni giuridiche differenziate all'interno della cittadinanza ${ }^{36}$.

\section{Conflitti di cittadinanza}

Questa tendenza, che i migranti esemplificano nel modo più efficace (e spesso drammatico), gioca un ruolo essenziale nel processo di costituzione materiale della cittadinanza europea e nello stesso funzionamento del mercato del lavoro nei diversi paesi europei, tanto che oggi il confine può essere considerato una delle leve fondamentali attorno a cui cittadinanza e mercato del lavoro si riorganizzano ${ }^{37}$. Si tratta di fenomeni spesso discussi nella letteratura sociologica sotto l'etichetta dell' «esclusione». Ho già più volte accennato al fatto che questa categoria, pur indubbiamente utile sotto il profilo descrittivo, rischia di risultare fuorviante se assunta nel suo significato letterale. Mi pare più corretto parlare, lo dicevo in precedenza, di inclusione differenziale.

Studiare il processo di formazione della cittadinanza europea assumendo come punto privilegiato di osservazione i suoi confini consente anzi di cogliere le profonde trasformazioni che stanno investendo la semantica e le forme dell'inclusione. Per quanto le politiche di controllo dei confini esterni dell'Unione europea si siano in questi anni organizzate retoricamente attorno all'obiettivo di bloccare i movimenti di rifugiati e profughi, il loro effetto non è stato in alcun modo quello di sigillare ermeticamente i confini. Più che alla costruzione delle mura di una «fortezza», si è piuttosto assistito alla predisposizione di un sistema di «dighe», di meccanismi di «filtraggio» e di governo selettivo della mobilità ${ }^{38}$. Analogamente a quel che è stato osservato a proposito del confine tra Stati uniti e Messico, si può affermare che le politiche di controllo dei confini esterni europei abbiano finito con il determinare «un

36. Cfr. Balibar, E. Noi cittadini d'Europa? Le frontiere, lo Stato, il popolo (2001). Roma: Manifestolibri, 2004.

37. Cfr. Raimondi, F.; Ricciardi, M. (ed.), Lavoro migrante. Esperienza e prospettiva. Roma: Derive Approdi, 2004.

38. Cfr. in questo senso il bel saggio di WALTERS, W. «Secure Borders, Safe Haven, Domopolitics». Citizenship Studies, 8, 2004, p. 237-260. 
processo attivo di inclusione del lavoro migrante attraverso la sua clandestinizzazione» ${ }^{39}$.

Quel che vorrei conclusivamente aggiungere, tuttavia, è che un'analisi della cittadinanza (in generale, ma anche in particolare in riferimento alla cittadinanza europea) non può limitarsi alla sua definizione giuridico-istituzionale. Le ricerche degli ultimi anni, sotto il profilo storico, sociologico, politologico, ci insegnano piuttosto a considerare decisivo per la considerazione della cittadinanza un insieme di pratiche sociali, di movimenti e di comportamenti soggettivi che, pur inscrivendosi all'interno del perimetro istituzionale della cittadinanza stessa, possono metterlo in discussione, in particolare forzandone $\mathrm{i}$ confini. Da questo punto di vista, gli stessi movimenti migratori contemporanei possono essere considerati come attraversati e costituiti da un insieme complesso di domande soggettive di cittadinanza che quotidianamente contestano i confini della cittadinanza europea. E la cittadinanza torna così a essere, al di là di ogni illusoria pacificazione, uno spazio di conflitto.

Sandro Mezzadra insegna «Studi coloniali e postcoloniali» e «Frontiere della cittadinanza» nella Facoltà di Scienze politiche dell'Università di Bologna. Si è occupato di storia delle scienze giuridiche, politiche e sociali tedesche tra Otto e Novecento e di autori classici della storia delle dottrine politiche (Hobbes, Spinoza, Marx). Negli ultimi anni ha lavorato in modo particolare su temi di teoria politica contemporanea, lavorando sul rapporto tra "globalizzazione», movimenti migratori e cittadinanza nonché sulle problematiche del «postcolonialismo». Fa parte della redazione di numerose riviste, tra cui «Filosofia politica», "Scienza \& Politica», «Studi culturali» e «Multitudes». Tra le sue pubblicazioni: La costituzione del sociale. Il pensiero politico e giuridico di Hugo Preuss, Bologna, Il Mulino, 1999 e Diritto di fuga. Migrazioni, cittadinanza, globalizzazione, Verona, Ombre corte, 2001. Ha recentemente curato, insieme a Furi Ferraresi, un'antologia degli scritti giovanili di Max Weber sulla questione agraria, Dalla terra alla fabbrica. Scritti sui lavoratori agricoli e lo Stato nazionale, Laterza, Roma, Bari, 2005.

39. Cfr. Genova, N. P. de. "Migrant «Illegality" and Deportability in Everyday Life». Annual Review of Anthropology, 31, 2001, p. 419-447. 